\title{
Electrowetting on conductors: Anatomy of the phenomenon
}

DOI:

10.1039/C6FD00252H

\section{Document Version}

Accepted author manuscript

Link to publication record in Manchester Research Explorer

\section{Citation for published version (APA):}

Ounnunkad, K., Patten, H., Velicky, M., Farquhar, A., Brooksby, P. A., Downard, A. J., \& Dryfe, R. (2017).

Electrowetting on conductors: Anatomy of the phenomenon. Faraday Discussions.

https://doi.org/10.1039/C6FD00252H

\section{Published in:}

Faraday Discussions

\section{Citing this paper}

Please note that where the full-text provided on Manchester Research Explorer is the Author Accepted Manuscript or Proof version this may differ from the final Published version. If citing, it is advised that you check and use the publisher's definitive version.

\section{General rights}

Copyright and moral rights for the publications made accessible in the Research Explorer are retained by the authors and/or other copyright owners and it is a condition of accessing publications that users recognise and abide by the legal requirements associated with these rights.

\section{Takedown policy}

If you believe that this document breaches copyright please refer to the University of Manchester's Takedown Procedures [http://man.ac.uk/04Y6Bo] or contact uml.scholarlycommunications@manchester.ac.uk providing relevant details, so we can investigate your claim.

\section{OPEN ACCESS}




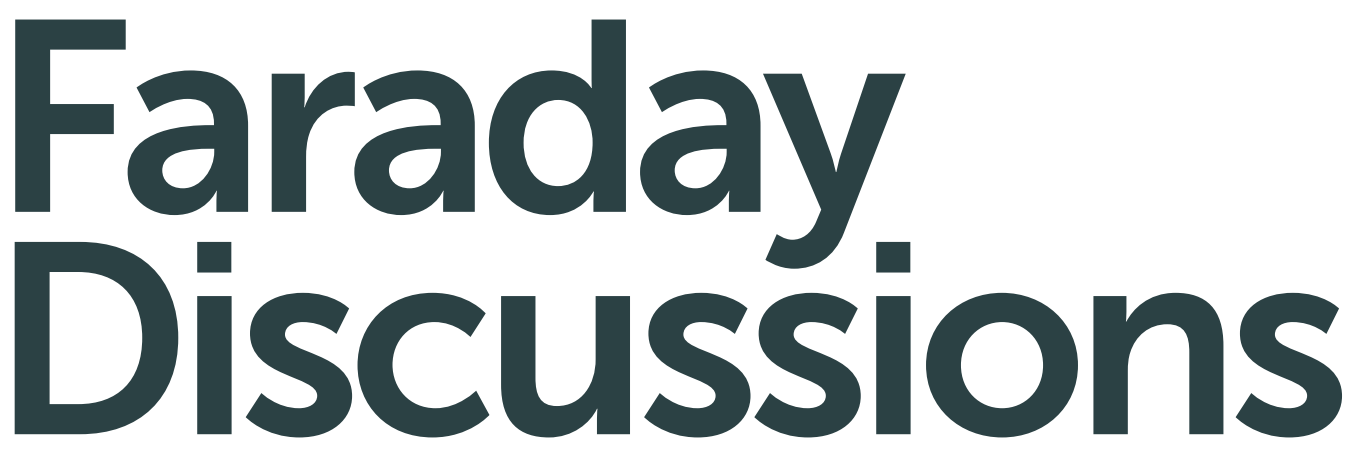

\section{Accepted Manuscript}

This manuscript will be presented and discussed at a forthcoming Faraday Discussion meeting.

All delegates can contribute to the discussion which will be included in the final volume.

Register now to attend! Full details of all upcoming meetings: http://rsc.li/fd-upcoming-meetings

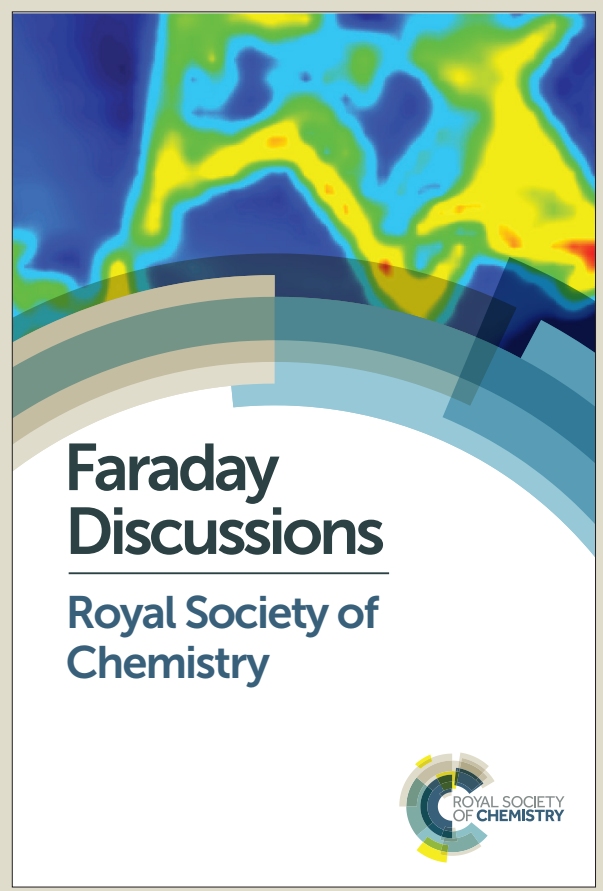

This is an Accepted Manuscript, which has been through the Royal Society of Chemistry peer review process and has been accepted for publication.

Accepted Manuscripts are published online shortly after acceptance, before technical editing, formatting and proof reading. Using this free service, authors can make their results available to the community, in citable form, before we publish the edited article. We will replace this Accepted Manuscript with the edited and formatted Advance Article as soon as it is available.

You can find more information about Accepted Manuscripts in the Information for Authors.

Please note that technical editing may introduce minor changes to the text and/or graphics, which may alter content. The journal's standard Terms \& Conditions and the Ethical guidelines still apply. In no event shall the Royal Society of Chemistry be held responsible for any errors or omissions in this Accepted Manuscript or any consequences arising from the use of any information it contains.

This article can be cited before page numbers have been issued, to do this please use: K. Ounnunkad, H. V. Patten, M. Velicky, A. Farquhar, P. A. Brooksby, A. J. Downard and R. Dryfe, Faraday Discuss., 2017, DOI: 10.1039/C6FD00252H. 


\title{
Electrowetting on conductors:
}

\section{Anatomy of the phenomenon}

\author{
Kontad Ounnunkad, ${ }^{1,2}$ Hollie V. Patten, ${ }^{1}$ Matěj Velický, ${ }^{1}$ Anna K. Farquhar, ${ }^{3}$ Paula A. \\ Brooksby, ${ }^{3}$ Alison J. Downard ${ }^{3}$ and Robert A. W. Dryfe ${ }^{1,4 *}$
}

1 = School of Chemistry, University of Manchester, Oxford Road, Manchester M13 9PL, UK.

2 = Chiang Mai University, Faculty of Science, Dept. of Chemistry, Chiang Mai 50200, Thailand

3 = Canterbury University, Dept. of Chemistry, MacDiarmid Institute of Advanced Materials \& Nanotechnology, Private Bag 4800, Christchurch 8140, New Zealand.

4 = National Graphene Institute, University of Manchester, Oxford Road, Manchester M13 9PL, UK.

Corresponding author email: robert.dryfe@manchester.ac.uk

\begin{abstract}
We have recently reported that reversible electrowetting can be observed on the basal plane of graphite, without the presence of a dielectric layer, in both liquid/air and liquid/liquid configurations. The influence of carbon structure on the wetting phenomenon is investigated in more detail here: specifically, it is shown that the adsorption of adventitious impurities on the graphite surface, markedly suppresses the electrowetting response. Similarly, the use of pyrolysed carbon films, although exhibiting a roughness below the threshold previously identified as the barrier to wetting on basal plane graphite, does not give a noticeable electrowetting response, which leads us to conclude that specific interactions at the watergraphite interface as well as graphite crystallinity are responsible for the reversible response seen in the latter case. Preliminary experiments on mechanically exfoliated and chemical vapour deposition grown graphene are also reported.
\end{abstract}


1. Introduction: Understanding of wetting phenomena, on a molecular scale, bridges many topical challenges for contemporary chemical physics as it requires detailed knowledge of the role of surface structure and topography, of solution/surface interactions, and of droplet motion on a variety of time and length scales. Although a variety of approaches for controlling wetting have been advocated, ${ }^{1}$ electrowetting has consistently attracted the most attention because of its reversibility and the simplicity of the basic electrowetting configuration. ${ }^{2}$ In the classical approach to electrowetting, the reduction in contact angle is driven by the energy stored in the capacitor created at the electrode/solution interface.

Charge storage requires the presence of ions on the solution side of the interface, although the requirement for the substrate to be a conductor has been circumvented by the development of the electrowetting-on-dielectric (EWOD) configuration: this approach has dominated technological applications of electrowetting since its introduction ca. twenty five years ago. ${ }^{3}$ The EWOD approach, although counter-intuitive from an electrochemical perspective as it necessities the use of higher voltages, has the advantage of suppressing surface oxidation and other electrolysis phenomena which confounded earlier attempts to move away from mercury as a substrate for wetting. ${ }^{4,5}$ This innovation has led to the widespread adoption of EWOD as a technological platform, notably in adaptive optics and droplet actuation applications. ${ }^{6,7}$ Many of these applications of electrowetting require a dual liquid phase geometry, where the conducting electrolyte droplet (frequently an aqueous solution) is surrounded by an immiscible (normally, organic) liquid.

Over recent years, however, there have been a few reports that have dispensed with the dielectric layer, i.e. reverting to the classical electrowetting-on-conductor (EWOC) configuration. Notably, Kornyshev and co-workers described an EWOC approach based on the liquid/liquid interface where both liquid phases contain electrolytes, so-called electrochemistry at the interface between two immiscible electrolyte solutions (ITIES), ${ }^{8}$ giving rise to a well-defined double layer at the liquid-liquid interface. ${ }^{9-12}$ This permitted the 
experimental realisation, in collaboration with Kucernak, of a dielectric-free ITIES approach to electrowetting based on the use of gold as the substrate, where significant changes in droplet contact angle $\left(\approx 30^{\circ}\right)$ were obtained with applied potentials of less than $1 \mathrm{~V}$. $^{13}$ To obtain a flat substrate, and thereby minimise "pinning" of the droplet on surface steps, the gold film was prepared by sputtering on to a titanium-coated quartz substrate. Other groups have subsequently reported classical EWOC experiments, based on aqueous electrolyte phases surrounded by air. Pu et al used composite films of graphene and carbon nanotubes, which yielded large (up to $50^{\circ}$ ) changes in contact angle, although significant voltages were applied $( \pm 10 \mathrm{~V}) .{ }^{14}$ Liu et al employed a stainless steel substrate with an applied potential of $+1.5 \mathrm{~V}$, again observing a change in contact angle of ca. $50^{\circ}$, although the wetting effect was slow (minute timescale), polarity dependent and irreversible. ${ }^{15}$ Most recently, Unwin and coworkers have reported that reversible electrowetting occurs at positive potentials on graphite for aqueous solutions containing oxy-anions, with the phenomenon attributed to the reversible intercalation of these anions. ${ }^{16}$ Contact angle changes of ca. $25^{\circ}$, over $1 \mathrm{~V}$ potentials, were observed although considerable hysteresis was seen in the wettingdewetting response. A recent report from our laboratory has described reversible, hysteresis-free EWOC on basal plane graphite using concentrated aqueous electrolyte solutions, surrounded by air, organic solvents and exploiting the ITIES configuration proposed by Kornyshev et al. Contact angle changes of $50^{\circ}$, over a $1 \mathrm{~V}$ potential range, and $100^{\circ}$, over a $1.5 \mathrm{~V}$ potential range, were seen for the liquid/air and liquid/liquid configurations, respectively. ${ }^{17}$ The aim of the present contribution is to study this wetting on carbonaceous surfaces in more depth, noting that the interaction of water (and aqueous electrolyte solutions) with graphite, graphene and carbon nanotubes has been the focus of considerable activity over the past decade. This is driven by a desire to understand better the nature of the $\mathrm{sp}^{2}$ carbon-water interaction, given that such surfaces are believed to facilitate the motion of aqueous droplets over them, and is given a further impetus by numerous applications (e.g. in energy storage), where the wetting of small pores within graphitic carbons by electrolyte solutions is extremely important. ${ }^{18}$ Of further relevance to the 
current work is debate in the recent literature on the contact angle of graphene, specifically the degree to which the observed contact angle is dictated by the underlying substrate, ${ }^{19,20}$ and on the contact angle of graphite, including the extent to which ambient surface contaminants perturb the contact angle. ${ }^{21-23}$ The presence of the latter is also reported to affect the intrinsic electrochemical response of graphite surfaces, either through the effect on the kinetics of heterogeneous electron transfer, ${ }^{23-26}$ or on the capacitance of graphite/electrolyte interfaces. ${ }^{27}$ Here we describe the effect of surface treatment, including ambient ageing, on the graphite electrowetting response and also attempt to generalise the phenomenon to other carbonaceous surfaces including graphene, with the aim of better understanding the specific electrode-electrolyte surface interactions responsible for the reversible wetting process.

\section{Experimental: Materials}

The electrolyte solution used, unless otherwise stated, was aqueous $\mathrm{LiCl}(6 \mathrm{M})$, the salt was obtained from Sigma-Aldrich (> 99.0\% purity). Ultra-pure water (18.2 $\mathrm{M} \Omega \mathrm{cm}$ resistivity, total organic content $2 \mathrm{ppb}$ ) was obtained from a "Milli-Q" Ultrafiltration system, supplied by Merck Millipore. Pt wire (0.10 mm diameter, 99.99+\%, Advent Research Materials) and polyestercoated Pt wire were used as quasi-reference and counter electrodes, respectively $(0.125$ mm diameter, 99.99\%, Goodfellow Cambridge Ltd, UK). The highly oriented pyrolytic graphite (HOPG, ZYA grade) was obtained from Tips Nano UK and cleaved mechanically using Scotch tape (3M) immediately prior to contact with the electrolyte droplet. Diazonium functionalisation of the graphite was achieved by leaving the basal plane surface in contact with a $20 \mathrm{mM}$ solution of 4-nitrobenzenediazonium tetrafluoroborate (Sigma-Aldrich) in acetonitrile, for 1 hour. ${ }^{28}$

Pyrolysed photoresist film (PPF) was prepared by pyrolysis of the commercial photoresists (PR, supplied by Clariant) listed below, and spin-coating on Si wafers. Si wafer substrates 
were cut into approximately $10 \mathrm{~mm} \times 10 \mathrm{~mm}$ samples and cleaned in acetone, methanol and iso-propanol baths by sonication for $30 \mathrm{~s}$ in each solvent, before being dried with $\mathrm{N}_{2}$. Three PR layers were then spin coated onto the Si wafers (thin - thick- thin PR polymers): AZ1518, AZ4620, and AZ1518. Each resist was spun at $4000 \mathrm{rpm}$ for $45 \mathrm{~s}$ and soft-baked at $110^{\circ} \mathrm{C}$ on a hot plate for $20 \mathrm{~min}$ in air. A final oven soft-bake followed, at $110^{\circ} \mathrm{C}$ for $1.5 \mathrm{hr}$, also in air. The pyrolysis protocol consisted of heating to $300^{\circ} \mathrm{C}$, in a forming gas atmosphere for 40 mins, heating from $300^{\circ} \mathrm{C}$ to $600^{\circ} \mathrm{C}$, in a forming gas atmosphere for $40 \mathrm{mins}$, then heating from $600^{\circ} \mathrm{C}$ to $900^{\circ} \mathrm{C}$ in a forming gas atmosphere for 30 mins, and finally heating from $900^{\circ} \mathrm{C}$ to $1100^{\circ} \mathrm{C}$ in a forming gas atmosphere for 1 hour. The samples were then allowed to cool to room temperature under forming gas overnight.

The PPF was cleaned with iso-propanol in a small ultrasonic cleaning bath for $15 \mathrm{~s}$ and then rinsed with isopropanol several times. The aqueous electrolyte contact angle (in air) of such PPF samples was found to lie between $60^{\circ}$ and $70^{\circ}$. For some samples, the PPF was subsequently exposed to an ambient heat treatment where it was heated to $100^{\circ} \mathrm{C}$ for 1 hour: this was found to increase the initial contact angle of the aqueous droplet on the PPF to $80^{\circ}$. A higher temperature, inert atmosphere treatment $\left(500^{\circ} \mathrm{C}\right.$ for 1 hour under argon) was also employed, although the initial contact angle was found to be the same as that for the air-heated sample.

Graphene samples were prepared by either micro-mechanical exfoliation of natural graphite (Naturgraphit, GmbH, Germany) or chemical vapour deposition (CVD). Natural graphene sample were made by repeated cleavage using cello-tape to obtain a flat and pristine graphite surface, which was pressed onto clean $\mathrm{Si} / \mathrm{SiO}_{2}$ wafers, previously washed by successive ultrasonication in acetone and iso-propanol, blow-dried by $\mathrm{N}_{2}$, and treated with $\mathrm{O}_{2}$ plasma to remove airborne adsorbates. The tape was dissolved in methyl iso-butyl ketone and the wafers were washed in iso-propanol, blow-dried with $\mathrm{N}_{2}$ and baked on a hotplate for a couple of minutes. A fresh flake surface was exposed by a final peel using the above tape. Flakes were identified using optical microscopy and electrical contact made, as 
described below. Chemical vapour deposition (CVD) graphene samples were supplied by Graphenea (San Sebastián, Spain) as films on $\mathrm{Si} / \mathrm{SiO}_{2}$ wafers. ${ }^{29}$ The graphene films were cleaned by soaking in chloroform for 1 day and then rinsed with iso-propanol several times. The resultant samples were left to dry in air. Connection to the carbonaceous working electrodes was made using a $0.25 \mathrm{~mm}$ diameter copper wire connected via $\mathrm{Ag}$ epoxy resin (supplied by RS Components).

\section{Apparatus}

The EWOC experiment used a configuration reported previously, where EWOC was performed with either an Autolab PGSTAT302N potentiostat (Metrohm Autolab, Utrecht, Netherlands) or an Ivium Compactstat (Ivium Technologies BV, Eindhoven, Netherlands) using a three electrode set-up, where the carbon materials acted as the working electrodes. In the standard electrowetting configuration, droplets were deposited on the carbon substrate using a microinjector (PV820 Pneumatic PicoPump) to expel the electrolyte solution from a micropipette (drawn from borosilicate capillaries with a Sutter P-97 Flaming/Brown Micropipette Puller to give a tip with inner and outer diameters of approximately 0.5 and $2 \mu \mathrm{m}$ respectively). Both the substrate and the micropipette were controlled using manual micro-positioners, so that the micropipette could be brought in close proximity to the surface. The micropipette also served as the electrolyte reservoir within which the auxiliary electrodes were located $\approx 3 \mathrm{~cm}$ from the conducting substrate. The droplet shape during electrowetting was determined from side-on images primarily captured using a CCD camera (Infinity, Lumenera) with the droplet backlit using an LED light source. The contact angle was then determined using a custom-written MATLAB ${ }^{\mathrm{TM}}$ programme, as detailed in our earlier report. ${ }^{17}$

Atomic force microscopy (AFM) images were collected in PeakForce QNM tapping mode with a Multimode8 (Bruker) using silicon nitride SNL-10 cantilevers. Image analysis was performed with Nanoscope Analysis (v1.6, Bruker). Raman spectroscopy (Renishaw inVia, 
$532 \mathrm{~nm}$ laser excitation) and X-ray photoelectron spectroscopy (Kratos Axis Ultra electron spectrometer with a monochromated Al K $\alpha$ source, $\mathrm{h} v=1486.6 \mathrm{eV}$ ) were also performed as further means to characterise the carbon materials.

3. Results: Fig. 1 shows the time dependent evolution of the aqueous electrolyte contact angle on the HOPG basal plane. If the droplet is not immediately placed in contact with the solid, its contact angle evolved rapidly from an initial value of ca. $66^{\circ}$ to approximately $80^{\circ}$, where the timescale of this change, ca. 20 minutes as shown in Figure 1(a), is consistent with the recent work of Li, Amemiya and Amadei et al. ${ }^{21-23}$ This agrees with previous explanations of this effect as being due to adsorption of ambient organic material on the graphitic surface. Fig.1(b) shows the evolution in droplet contact angle towards the positive limit of the potential window $(+0.6 \mathrm{~V})$ : the equilibrium contact angle starts at a much lower value (ca. $20^{\circ}$ ) at this potential, however the substrate shows the same increase in hydrophobicity if left in the ambient over time. The contact angle reaches a new equilibrium value of ca. $60^{\circ}$ over a 4 hour timescale. Interestingly, contamination of the HOPG substrate is associated with an increase in the water contact angle, whereas heating of the PPF (see Experimental section), which is assumed to remove not only contaminants from the surface but also change the termination of surface, is seen to increase the water contact angle.

The increase in aqueous contact angle, for charged and uncharged surfaces depending on the degree of ambient exposure, is also reflected in differences in the potential-dependent contact angle, i.e. the electrowetting response, for freshly cleaved and "aged" HOPG surfaces, shown in Fig. 2. It is clear from the consistency in the wetting responses for the samples aged for 14 hours and 20 hours, that a new surface equilibrium is reached by the time 14 hours have elapsed. The wetting response of these aged surfaces is strongly suppressed, with a higher threshold potential (by approximately $0.4 \mathrm{~V}$ ) required to induce significant wetting and to achieve a considerable decrease (from $40^{\circ}$ to $20^{\circ}$ ) in the maximum 
contact angle change, compared to the "fresh" surface. These findings are consistent with other surface sensitive measurements on basal plane HOPG, for example, the effect of ambient exposure on its contact angle at neutrality, ${ }^{23}$ and our own recent work, which has shown that the capacitance of the surface is reduced by approximately $50 \%$ on exposure to the ambient. ${ }^{27}$ The reduction in capacitance was accompanied by a positive shift of the potential of zero charge (PZC), which was also observed in this study. The change in capacitance is also evident from the reduction in strength of the electrowetting response, which can be more clearly seen if the cosine of the droplet contact angle, $\theta$, is plotted vs. the square of the applied potential, $E$, following the Young-Lippmann equation (equation 1, and Fig. 2(b)): ${ }^{2}$

$$
\cos \theta=\cos \theta_{e q}+\gamma_{L V}^{-1} \int_{E_{p z c}}^{E} C(E) E d E \approx \frac{C\left(E-E_{p z c}\right)^{2}}{2 \gamma_{L V}}=\eta
$$

Where $C(E)$ denotes the potential-dependent capacitance of the solid/liquid interface (HOPG/aqueous $\mathrm{LiCl}$ in this case), $E_{\mathrm{pzc}}$ is the potential of zero charge, $\gamma_{\mathrm{Lv}}$ is the surface tension between the two fluid phases (aqueous/air here, taken to be $83.3 \mathrm{mN} \mathrm{m}^{-1}$ ) ${ }^{17}$, and $\eta$ denotes the electrowetting number. The analytical form of the expression results when the capacitance is assumed to be constant, an approximation made widely in the literature. ${ }^{2}$ The dynamics of the electrowetting response are not the specific focus of this work, but a preliminary investigation shows that the wetting dynamics are also sensitive to the ageing of the graphite, with a slowing of the time-scale of the response from ca. $10 \mathrm{~ms}$ to approaching 100 ms (see Fig 2(c)).

As well as the finding that the wetting is sensitive to the surface state of the graphite sample, we have also found that the wetting depends more generally on the type of carbon substrate used. The electrowetting response, for the same electrolyte droplet and potential range, of basal plane HOPG and thermally pre-treated PPF is shown in Fig 3, highlighting the lack of wetting response seen on the latter surface. A similar "null" response was obtained from the 
non-heated PPF sample, which has a lower initial contact angle. The lack of wetting response on the PPF cannot be attributed to a significant change in the electrochemical behaviour of this material. The response of PPF is compared to HOPG (see Supporting Information, Fig. SI1), showing that the most significant change in electrochemical response occurs on ageing of the graphite sample, particularly at positive potentials. Similarly, Fig. SI2 shows the plan-view optical micrographs of the aqueous droplets, again showing no significant deviation from the ideal spherical cap geometry for the droplets injected onto largely defect-free regions of the HOPG, PPF and CVD graphene (discussed further below). AFM of the PPF samples does, however, reveal a greater surface roughness than basal plane HOPG, as shown in Fig. 4(a) and (b), which present a few isolated larger particles on the PPF. In spite of the presence of the larger particles, the surface roughness is still below the threshold of $100 \mathrm{~nm}$ previously observed to cause droplet pinning on graphite surfaces. ${ }^{17}$ Raman spectroscopy is also presented in Fig. 4 and is consistent with previous reports for this material. ${ }^{30}$ The characterisation presented within Fig. 4 for the graphitic surface is also consistent with literature reports for this material. XPS analysis (data not shown) indicates that the primary effect of ageing on composition is an increase in oxygen content from ca. $1 \%$ for the freshly cleaved surface to ca. $3 \%$ for the ambient aged surface. We therefore interpret the lack of wetting seen for PPF as evidence for a specific interaction between aqueous solutions and graphitic (i.e. exclusively $\mathrm{sp}^{2}$ carbon) surfaces. This is related to recently observed phenomena such as the ultra-rapid transport of water through carbon nanotubes and precision "nano-capillaries" fabricated between graphite flakes using graphene "spacers" as the capillary walls. ${ }^{31,32}$ PPFs can be viewed as "disordered" graphitic materials, which, despite their relatively low surface roughness, are believed to contain a higher number of $\mathrm{H}$-terminated (i.e. $\mathrm{sp}^{3}$ carbon atoms) at their surface. ${ }^{30}$

Further support for the hypothesis that a specific low-friction interaction between the "unsaturated" carbon surface and aqueous solution is responsible for the low-voltage wetting phenomenon is obtained by forming $\mathrm{sp}^{3}$ hybridised carbon atoms on the graphite surface via 
diazonium functionalisation, following the literature treatment described above (see Experimental section). This treatment transforms a proportion of the basal plane carbon atoms to an $\mathrm{sp}^{3}$ hybridised state through grafting of the reactive aryl radical on to the graphite surface. ${ }^{33}$ Ideally, only a low density monomer coverage is obtained, but the high reactivity of the aryl intermediate means that oligomer structures also form, increasing the surface roughness. ${ }^{28}$ The difference in roughness before, and after, the diazonium treatment is evident from the AFM analyses presented in Fig 5 (a) and (b), respectively.

Functionalisation is also demonstrated from the voltammetric response in Fig 5(c), which reveals new reductive features associated with the presence of the nitro group, ${ }^{28}$ and the Raman spectroscopy, Fig. 5(d), showing the appearance of a D peak due to the formation of sp3 "defects" within the graphite lattice, and the small upshift of the G-band (ca. $1 \mathrm{~cm}^{-1}$ ), associated with p-doping of the substrate due to the presence of the nitro group. ${ }^{34}$ The surface coverage of the nitrophenyl moieties (i.e. $\mathrm{sp}^{3}$ carbon) can be estimated from the voltammetric response to be $0.47 \mathrm{~nm} \mathrm{~cm}^{-2}$, assuming a two electron re-oxidation of the aryl group occurs. ${ }^{35}$ Signficantly, no wetting response was observed within the potential window used for the other carbon substrates (see Supporting information), however regeneration of the "fresh" HOPG surface by peeling of the uppermost layers, recovered the electrowetting response.

We have also compared the response seen on the "bulk" graphite surface, by studying wetting on its 2-dimensional analogue, i.e. by using graphene as the conducting substrate. In fact, no significant wetting response was seen for CVD graphene, see Fig. 6 , an effect that may be attributed to the presence of residual polymer particles from the substrate transfer process as well as high polycrystallinity of CVD graphene. ${ }^{36-38}$ The AFM of the CVD graphene shows visible grain boundaries, Fig. 6(b), compared to the graphite and PPF samples. By contrast, "top-down" images of electrolyte droplets on various thicknesses of mechanically-exfoliated graphene do show reversible wetting responses, see video files uploaded as Supporting Information, which are consistent with the behaviour observed for 
basal plane graphite. The electrowetting effect is seen as a series "pulses" in the droplet size on the mechanically exfoliated graphene, is more pronounced at positive potentials. This asymmetry has been noted previously for the $\mathrm{LiCl}$ electrolyte on $\mathrm{HOPG}^{17}$ although this asymmetry becomes less pronounced as the thickness of the sample is reduced (See Supporting videos). The observation of asymmetry suggests a more repulsive interaction occurs between the substrate and the halide ions, in excess at positive potentials, which facilitates the "minimum slip" movement of the aqueous droplet over the graphite surface. In contrast, a more strongly bound aqueous network results at negative potentials, when the lithium cations dominate the electrical double layer. Such asymmetry, due to specific graphite-electrolyte interaction, is weakened as the conducting substrate becomes thinner, and as interactions with the underlying $\mathrm{Si} / \mathrm{SiO}_{2}$ substrate start to influence the overall wetting response. The extent of "contact angle transparency" for graphene layers on insulating substrates is a topic of recent debate..$^{19,20}$

\section{Conclusions}

Reversible electrowetting is demonstrated on basal plane graphite, although the magnitude of the effect is susceptible to ageing, with ambient contamination believed to be responsible for this phenomenon. No significant electrowetting effect is observed, under the conditions employed here, for PPF and CVD graphene samples, or for the case of highly defective graphite surfaces, formed through diazonium functionalisation. The failure to observe wetting in the PPF and nitrophenyl-functionalised cases suggests that a specific "low-friction" chemical interaction and lack of inhibition by grain boundaries is responsible for the wetting seen on graphite. Further work remains, however, to resolve the "physical" and "chemical" contributions to the wetting, since the PPF and nitrophenyl-functionalised surfaces, as well as having a lower density of $\mathrm{sp}^{2}$ carbon atoms, are also slightly rougher than the original HOPG substrate. In contrast, the observation of wetting on mechanically exfoliated 
graphene suggests that the lack of wetting on CVD graphene is caused by the presence of transfer debris on the sample surface, causing pinning of droplets, although it is possible that grain boundaries also play a role in the CVD case. Importantly, the observation of wetting on the basal plane of graphene points against recently proposed ion intercalation as the modus operandus of the wetting process. ${ }^{16}$ This study offers some guidance to the future development of strategies for the observation of electrowetting on conductors, which present a low voltage, low hysteresis alternative to the widely adopted electrowetting on a dielectric approach. Further studies, particularly of the dynamics of the wetting process, are in progress.

Acknowledgements

K.O. acknowledges support from the Newton Fund (Travel Grant-Research Links 2016). R.D. thanks the EPSRC (grant ref. EP/K016954/1) for support. A.K.F. thanks the Royal Society of New Zealand Marsden Fund (UOC1307), for supporting her visit to Manchester.

References:

1. J. A. Lv, Y. Liu, J. Wei, E. Chen, L. Qin and Y. Yu, Nature, 2016, 537, 179.

2. F. Mugele, Soft Matter, 2009, 5, 3377.

3. B. Berge, Comp. Rend. Acad. Sci. Ser. II, 1993, 317, 157.

4. A. Frumkin, A. Gorodetzkaja, B. Kabanow and N. Nekrassow, Phys. Z. Sovijetunion., 1933, 1, 255.

5. M. J. Sparnaay, Surf. Sci., 1964, 1, 213. 
6. S. Kuiper and B.H.W Hendriks, Appl. Phys. Lett., 2004, 85, 1128.

7. A. R. Wheeler, Science, 2008, 322, 539.

8. F. Reymond, D. J. Fermín, H. J. Lee and H. H. Girault, Electrochim. Acta, 2000, 45, 2647.

9. C. W. Monroe, L. I. Daikhin, M. Urbakh and A. A. Kornyshev, Phys. Rev. Lett., 2006, 97, 136102.

10. C. W. Monroe, M. Urbakh and A. A. Kornyshev, J. Phys. Cond. Matter, 2007, 19, 375113.

11. C. W. Monroe, M. Urbakh and A. A. Kornyshev, J. Electrochem. Soc., 2009, 156, P21.

12. M. Marinescu, T. Barnea, M. Urbakh and A.A. Kornyshev, J. Phys. Chem. C, 2010, 114, 22558.

13. A. A. Kornyshev, A. R. Kucernak, M. Marinescu, C. W. Monroe, A. E. S. Sleightholme, and M. Urbakh, J. Phys. Chem. C, 2010, 114, 14885.

14. J. Pu, S. Wan, Z. Lu, G. A. Zhang, L. Wang, X. Zhang and Q. J. Xue, J. Mater. Chem. A, 2013,1, 1254.

15. Y. Liu, Y. E. Liang, Y. J. Sheng and H. K. Tsao, Langmuir, 2015, 31, 3840.

16. G. Zhang, M. Walker and P. R. Unwin, Langmuir, 2016, 32, 7476.

17. D. J. Lomax, P. Kant, A. T. Williams, H.V. Patten, Y. Zou, A. Juel and R. A. W. Dryfe,

Soft Matter, 2016, 12, 8798.

18. J. Chmiola, G. Yushin, Y. Gogotsi, C. Portet, P. Simon and P. L. Taberna, Science, $2006,313,1760$.

19. J. Rafiee, X. Mi, H. Gullapalli, A. V. Thomas, F. Yavari, Y. Shi, P. M. Ajayan and N. A. Koratkar, Nature Materials, 2012, 11, 217.

20. C. J. Shih, Q.H. Wang, S. Lin, K. C. Park, Z. Jin, M. S. Strano and D. Blankschtein, 
21. Z. Li, Y. Wang, A. Kozbial, G. Shenoy, F. Zhou, R. McGinley, P. Ireland, B.

Morganstein, A. Kunkel, S.P. Surwade, L. L and H. Liu, Nature Mater. 2013, 12, 925.

22 C.A. Amadei, C.Y. Lai, D. Hesker and M. Chiesa, J. Chem. Phys., 2014, 141, 084709.

23. Z.T. Li, A. Kozbial, N. Nioradze, D. Parobek, G. J. Shenoy, M. Salim, S. Amemiya, L. Li and H. T. Liu, ACS Nano, 2016, 10, 349.

24. A. S. Cuharuc, G. Zhang and P. R. Unwin, Phys. Chem. Chem. Phys., 2016, 18, 4966.

25. M. Velický, D. F. Bradley, A. J. Cooper, E. W. Hill, I. A. Kinloch, A. Mishchenko, K. S. Novoselov, H. V. Patten, P. S. Toth, A. T. Valota, S. D. Worrall and R. A. W. Dryfe, ACS Nano, 2014, 8, 10089.

26. M. Velický, M. A. Bissett, P. S. Toth, H. V. Patten, S. D. Worrall, A. N. J. Rodgers, E. W. Hill, I. A. Kinloch, K. S. Novoselov, T. Georgiou, L. Britnell and R. A. W. Dryfe, Phys. Chem. Chem. Phys., 2015, 17, 17844.

27. Y. Zou, A. S. Walton, I. A. Kinloch and R. A. W. Dryfe, Langmuir, 2016, 32, 11448.

28. T. Menanteau, E. Levillain and T. Breton, Langmuir, 2014, 30, 7913.

29. S. Bae, H. Kim, Y. Lee, X. Xu, J. S. Park, Y. Zheng, J. Balakrishnan, T. Lei, H. R. Kim, Y. I. Song, Y. J. Kim, K. S. Kim, B. Özyilmaz, J. H. Ahn, B. H. Hong and S. lijima, Nature Nanotech, 2010, 5, 574.

30. R.L. McCreery, Chem. Rev., 2008, 108, 2646.

31. E. Secchi, S. Marbach, A. Nigues, D. Stein, A. Siria and L. Bocquet, Nature, 2016, 537, 210. 
32. B. Radha, A. Esfandiar, F. C. Wang, A. P. Rooney, K. Gopinadhan, A. Keerthi, A. Mishchenko, A. Janardanan, P. Blake, L. Fumagalli, M. Lozada-Hidalgo, S. Garaj, S. J. Haigh, I. V. Grigorieva, H. A. Wu and A. K. Geim, Nature, 2016, 538, 222.

33. J. Lehr, B. E. Williamson and A. J. Downard, J. Phys. Chem. C, 2011, 115, 6629.

34. Q. H. Wang, Z. Jin, K. K. Kim, A. J. Hilmer, G. L. C. Paulus, C. J.Shih, M. H. Ham, J. D. Sanchez-Yamagishi, K. Watanabe, T. Taniguchi, J. Kong, P. Jarillo-Herrero and M. S. Strano, Nature Chem., 2012, 4, 724.

35. P. A. Brooksby, J. D. Shields, A. K. Farquhar and A. J. Downard, ChemElectrochem, 2016, 3, 2021.

36. H.V. Patten, M. Velický, N. Clark, C. A. Muryn, I. A. Kinloch and R. A. W. Dryfe, Faraday Disc., 2014,172, 261.

37. H. I. Rasool, C. Ophus and A. Zettl, Adv. Mater., 2015, 27, 5771.

38. P. Nemes-Incze, P. Vancsó, Z. Osváth, G. I. Márk, X. Jin, Y. S. Kim, C. Hwang, P. Lambin, C. Chapelier and L. P. Biró, Carbon, 2014, 66, 743.

Figure captions:

Figure $1-$ a) Contact angle evolution on HOPG at the PZC as a function of time after cleavage $(-0.45 \mathrm{~V})$, b) Contact angle evolution on HOPG an applied potential of $+0.6 \mathrm{~V}$ as a function of time after cleavage.

Figure 2 - a) Static electrowetting response of $6 \mathrm{M} \mathrm{LiCl(aq.)} \mathrm{droplets} \mathrm{on} \mathrm{fresh} \mathrm{(black}$ squares) and aged (14 hrs - blue circles, 20 hrs - red diamonds) basal plane HOPG. b) the 
data shown in a) presented as the cosine of the contact angle as a function of the square of the displacement in potential from the PZC for each sample. The PZC is taken to be $-0.45 \mathrm{~V}$ for fresh HOPG and $-0.30 \mathrm{~V}$ for the aged material. c) Time-dependent of the wetting response for fresh (black) and aged (red) HOPG on stepping the potential from the PZC of each sample to $+0.6 \mathrm{~V}$. The latter sample was aged for 3 days.

Figure 3 - Static electrowetting response of $6 \mathrm{M} \mathrm{LiCl(aq.)} \mathrm{droplets} \mathrm{on} \mathrm{fresh} \mathrm{basal} \mathrm{plane}$ HOPG (black squares) and PPF (red diamonds).

Figure $4-$ a) Characterisation of PPF: Peak force AFM map, profile along the line indicated in map and Raman spectrum. b) Characterisation of HOPG: Peak force AFM map, profile along the line indicated in map and Raman spectrum.

Figure $5-$ a) Peak force AFM image and line profile along the dotted line shown of the HOPG sample prior to diazonium functionalisation. b) Peak force AFM image and line profile along the dotted line shown of the HOPG sample following diazonium functionalisation. c) Voltammetric response for the diazonium-functionalised sample at a scan rate of $0.05 \mathrm{~V} \mathrm{~s}^{-1}$, in a $6 \mathrm{M} \mathrm{LiCl(aq.)} \mathrm{electrolyte} \mathrm{solution,} \mathrm{d)} \mathrm{Raman} \mathrm{spectrum} \mathrm{of} \mathrm{the} \mathrm{material} \mathrm{post-}$ functionalisation.

Figure 6 -a)“Side-on” images of $6 \mathrm{M} \mathrm{LiCl(aq.)} \mathrm{droplets} \mathrm{on} \mathrm{CVD} \mathrm{graphene} \mathrm{at} \mathrm{various} \mathrm{applied}$ potentials. b) peak force AFM image of the same sample and line profile along the dotted line shown. c) Raman spectroscopy of the CVD graphene sample. 

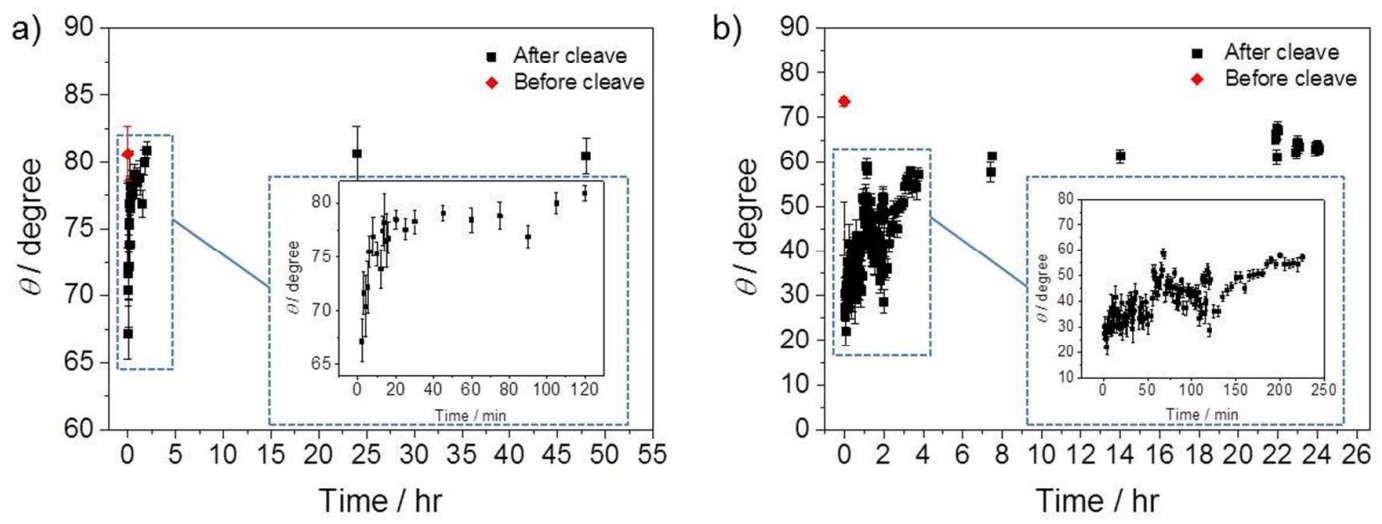

Fig. 1 

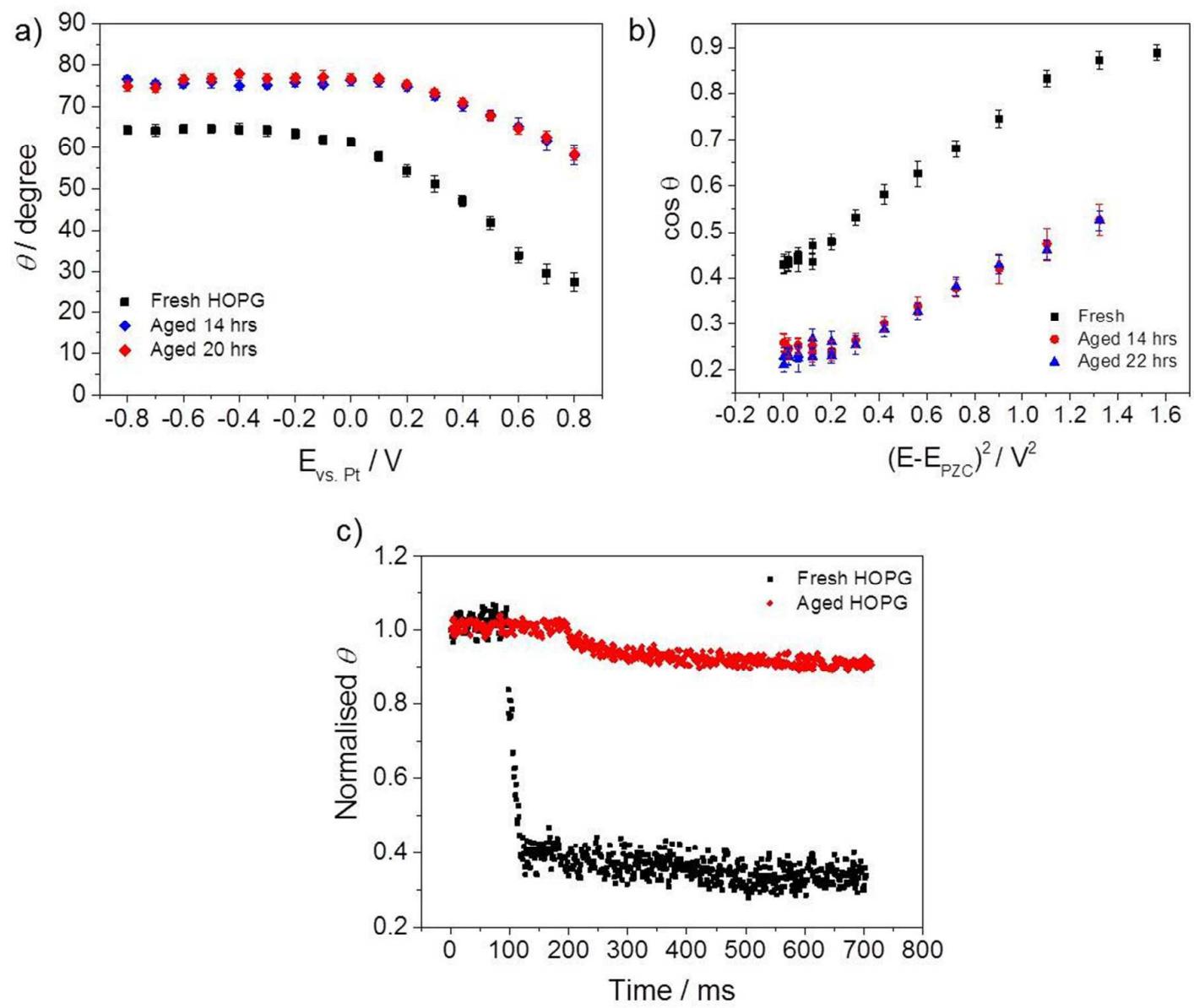

Fig. 2 


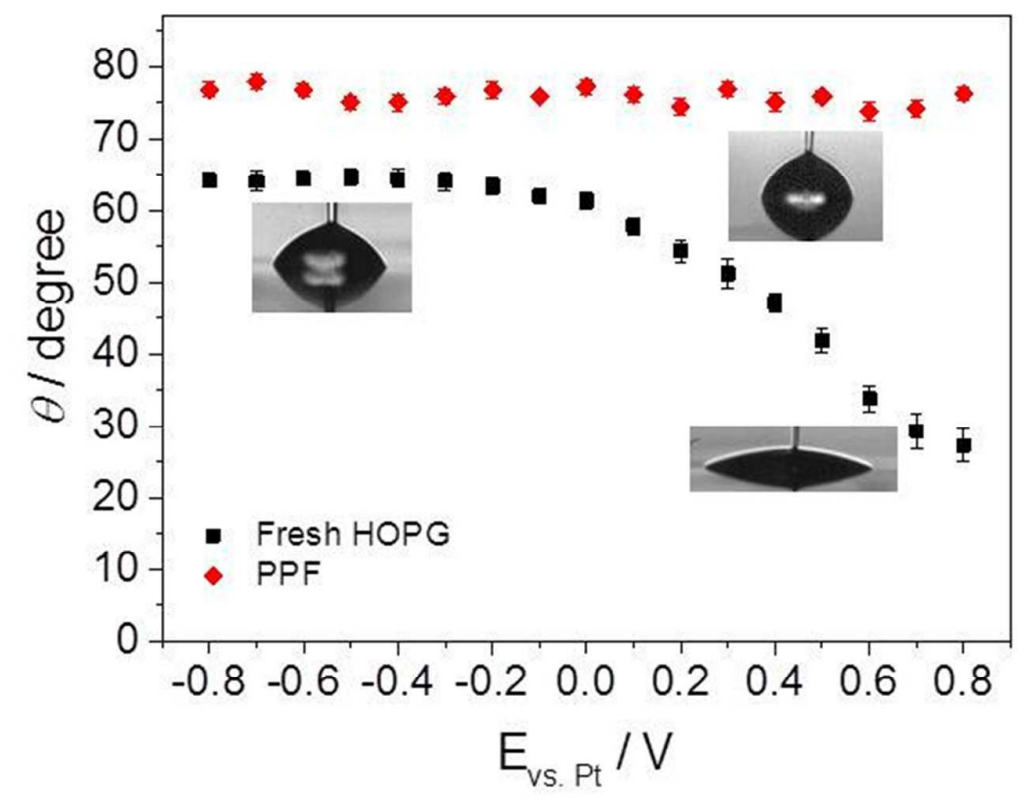

Fig. 3 

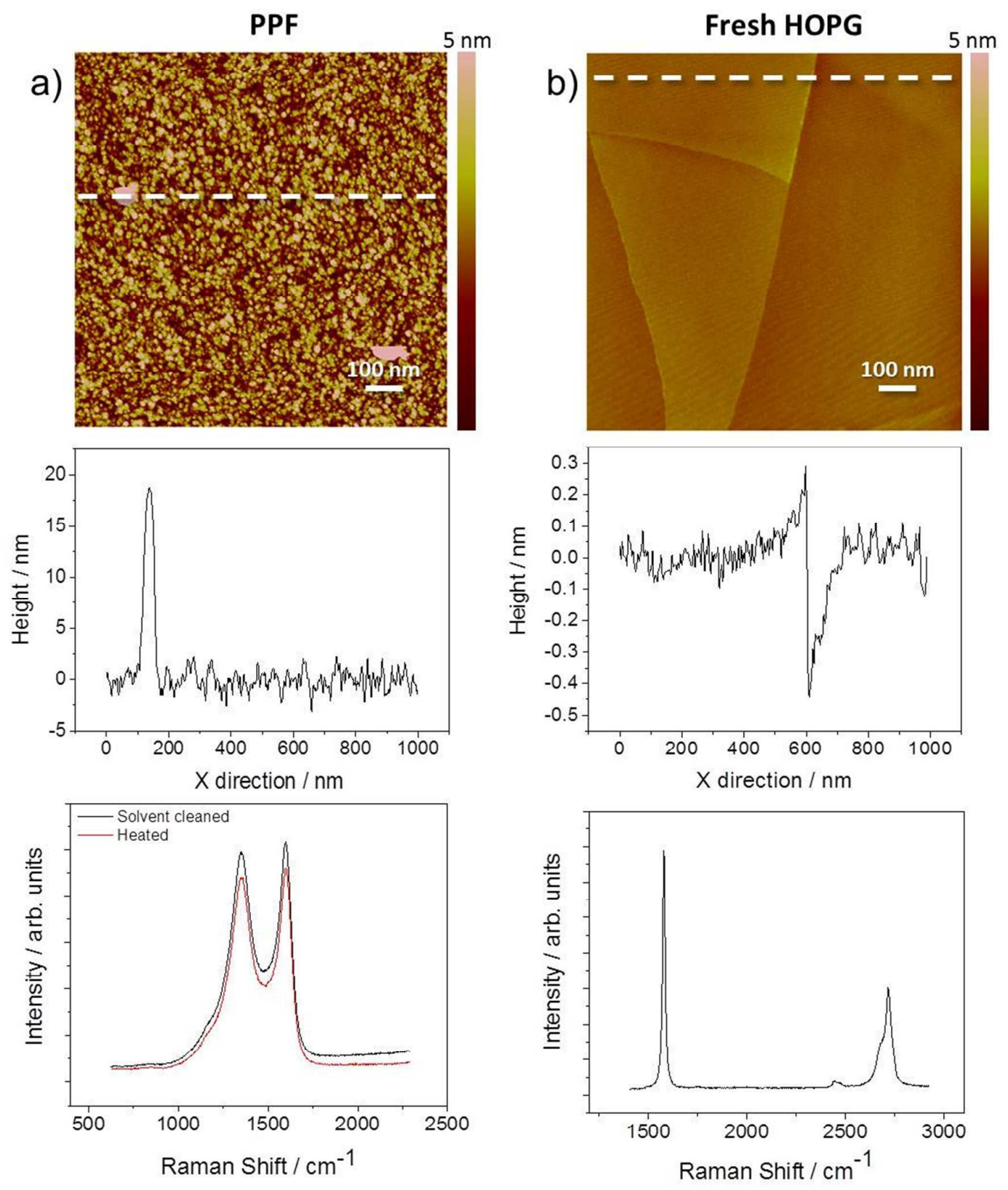

Fig. 4 
a)

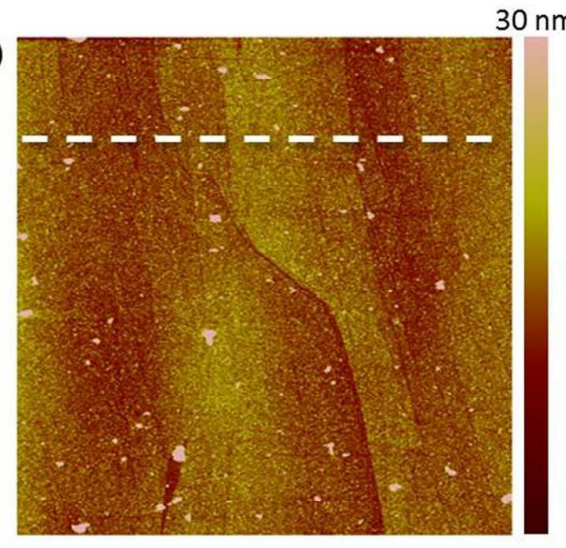

b)
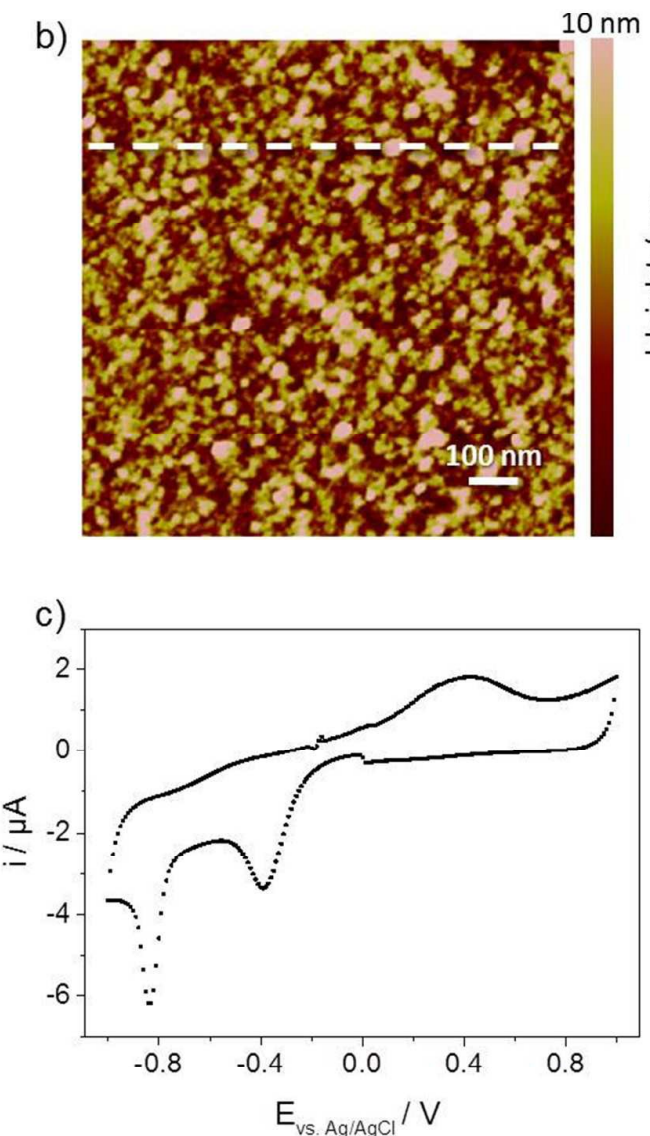
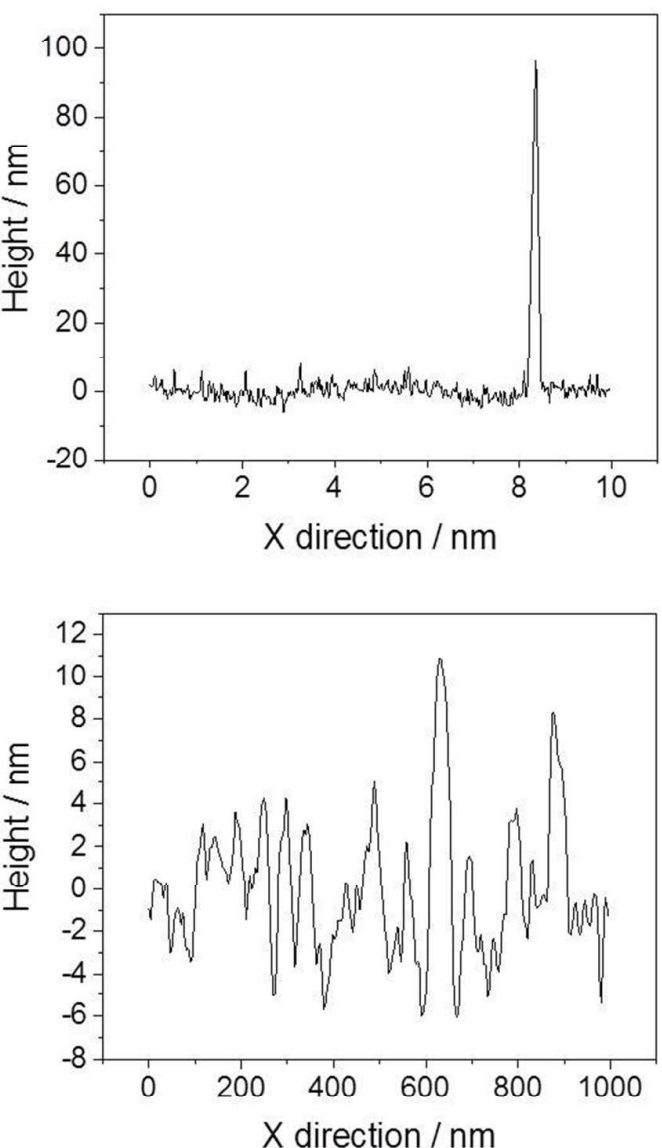

d)

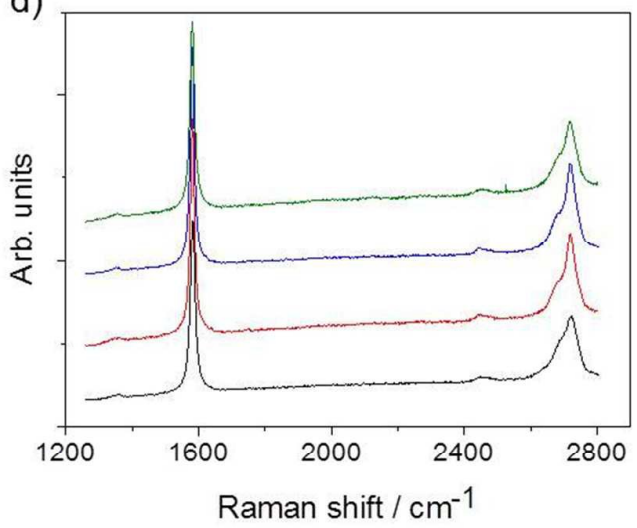

Fig. 5 

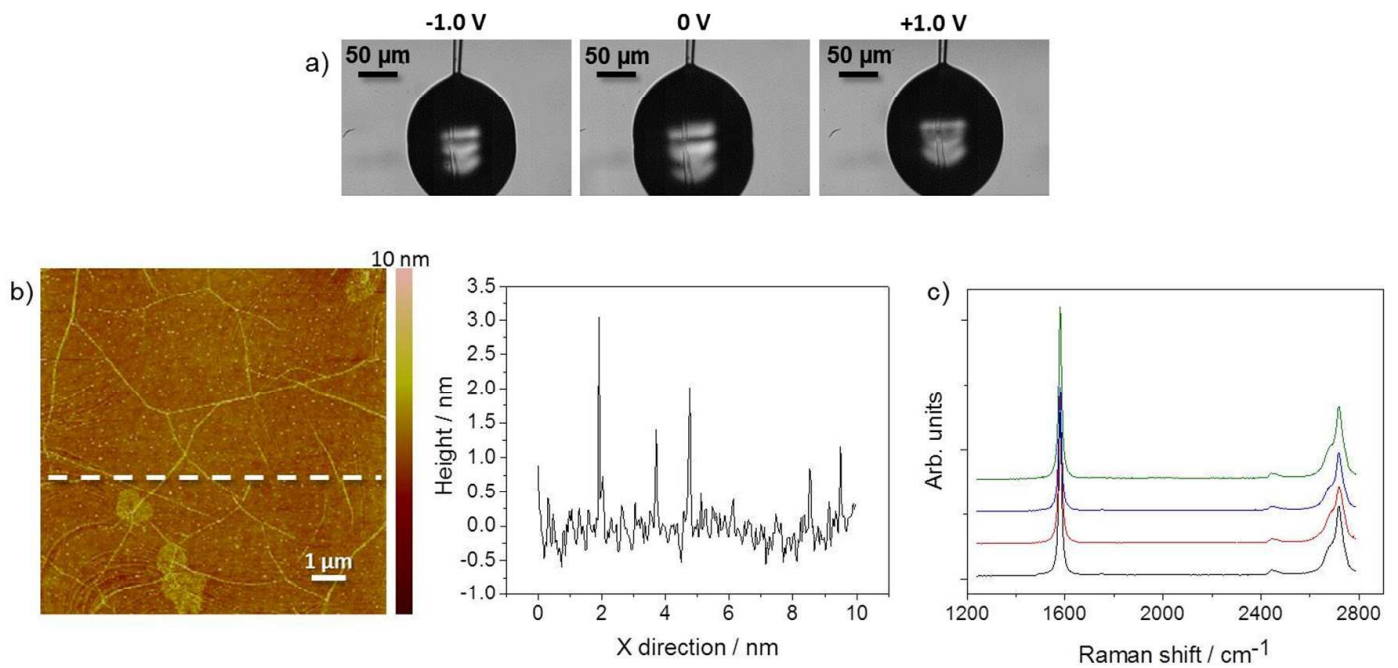

Fig. 6 